Henrique Leonardo Guerra ${ }^{1}$

Sandhi Maria Barreto 1,2

Elizabeth Uchôa 1,3

Josélia Oliveira Araújo Firmo 1

Maria Fernanda Furtado de Lima e Costa 1,2

\section{A morte de idosos na Clínica Santa Genoveva, Rio de Janeiro: um excesso de mortalidade que - sistema público de saúde poderia ter evitado}

\author{
Death of elderly patients in the Santa Genoveva \\ Clinic in Rio de Janeiro: excess mortality that \\ the public health system could have prevented
}

1 Laboratório

de Epidemiologia

e Antropologia Médica,

Centro de Pesquisas

René Rachou,

Fundação Oswaldo Cruz.

Av. Augusto de Lima 1715,

Belo Horizonte, $M G$

30190-002, Brasil.

2 Departamento

de Medicina Preventiva

e Social, Faculdade

de Medicina, Universidade

Federal de Minas Gerais.

Av. Alfredo Balena 190,

Belo Horizonte, $M G$

30130-100, Brasil.

3 Departamento

de Psiquiatria e Neurologia,

Faculdade de Medicina,

Universidade Federal

de Minas Gerais.

Av. Alfredo Balena 190,

Belo Horizonte, $M G$

30130-100, Brasil.

\begin{abstract}
From January to May 1996, 156 inpatients died in a clinic for elderly people in Rio de Janeiro, Brazil. The highest mortality rate was observed in May: 143/1,000 inpatients. As a result, the clinic was closed by the Ministry of Health. This study investigated whether the excessive number of deaths observed in the clinic in early 1996 was unexpected or reflected prevailing conditions. The investigation used the Public Health System database (SIH-SUS). The study period was 01/1993 to 05/1996. The investigation was based on: 1) a time-series analysis of the number of deaths and crude mortality rates and 2) comparison of the mortality rates observed in that clinic with those calculated for 15 area hospitals, defined as the reference rates. Risk of death in the clinic was higher than expected in 28 of the 41 months considered in the study. Highest risks were observed in January 1993 (RRcrude = 2.23; 95\% CI 1.56-3.14) and May 1996 (RRadjusted = 2.73; 95\% CI 1.88-3.95). The high mortality rates observed in the clinic in 1996 were already present in 1993. Thus, adequate use of the SIH-SUS could have anticipated and avoided the excess mortality identified in early 1996.
\end{abstract}

Key words Hospital Mortality; Hospital Records; Health Services for the Aged; Aged

Resumo Entre janeiro e maio de 1996, 156 idosos morreram na Clínica Santa Genoveva, no Rio de Janeiro. A mortalidade mais alta foi observada em maio: 143/1.000 internações. Isto resultou no fechamento da clínica pelo Ministério da Saúde. O objetivo deste trabalho é verificar, utilizando-se dados do Sistema de Informações Hospitalares do Sistema Único de Saúde (SIH-SUS), se os óbitos ocorridos na clínica em 1996 representavam uma exceção ou se refletiam condições já existentes. O periodo do estudo foi de janeiro/1993 a maio/1996. A metodologia da investigação incluiu 1) análise da série histórica do número e das taxas mensais brutas de mortalidade e 2) comparações destas com aquelas de 15 hospitais definidos como referência. O risco de morrer na clínica foi superior ao dos hospitais de referência em 28 dos 41 meses considerados. Os maiores riscos relativos foram observados em janeiro de 1993 (RRbruto $=2,23 ;$ IC-95\% $=1,56-3,14) e$ maio de 1996 (RRajustado = 2,73; IC-95\% = 1,88-3,95). Os resultados mostram que a alta mortalidade na clínica já vinha ocorrendo desde 1993. A utilização adequada do SIH-SUS poderia ter antecipado e evitado o excesso de mortalidade só identificado em meados de 1996.

Palavras-chave Mortalidade Hospitalar; Registros Hospitalares; Serviços de Saúde para Idosos; Idoso 


\section{Introdução}

A morte de elevado número de idosos internados na Clínica Santa Genoveva, Rio de Janeiro, entre os meses de abril e junho de 1996 foi amplamente denunciada pela imprensa. Isto resultou na intervenção do Ministério da Saúde, com subseqüente descredenciamento da clínica pelo Sistema Único de Saúde (SUS) e prisão preventiva de seis membros da sua direção (Folha de São Paulo, 1996). Estes fatos levam à reflexão a respeito da necessidade de o SUS monitorar a qualidade da assistência hospitalar dos serviços por ele contratados.

A taxa de mortalidade hospitalar bruta ou ajustada tem sido usada como indicador de qualidade da assistência hospitalar em países desenvolvidos (Roemer et al., 1968; DesHarnais et al., 1988; Hofer \& Hayward, 1996). No Brasil, apesar da existência de uma grande base de dados pública relativa a internações hospitalares (Sistema de Informações Hospitalares do Sistema Único de Saúde - SIH-SUS), a sua utilização para estudos epidemiológicos ainda é incipiente. Este fato é surpreendente, uma vez que, desde 1993, as informações acerca das internações hospitalares ocorridas no âmbito do SUS estão disponíveis em CD-ROM, não havendo restrições quanto ao seu uso (MS, 1998a).

O objetivo do presente trabalho é verificar, por intermédio dos dados do SIH-SUS, se os óbitos ocorridos na Clínica Santa Genoveva em 1996 representavam ocorrência pontual, ou se refletiam condições previamente existentes na clínica, situação em que seriam preveníveis mediante monitoramento adequado.

\section{Metodologia}

A fonte de dados para este trabalho foi o Sistema de Informações Hospitalares do Sistema Único de Saúde (SIH-SUS) (MS, 1998b). Neste, a Clínica Santa Genoveva foi localizada por meio de seu número no Cadastro Geral de Contribuintes (CGC). As internações hospitalares e os dados referentes às mesmas foram determinados através do número da Autorização de Internação Hospitalar (AIH), que identifica o paciente dos serviços prestados pelo SUS sob regime de internação hospitalar (MS, 1998a).

As internações analisadas referem-se àquelas ocorridas na Clínica Santa Genoveva e em quinze hospitais da cidade do Rio de Janeiro, utilizados para comparação (hospitais de referência). O período considerado para estudo foi de 01/01/1993 a 31/05/1996, datas que correspondem respectivamente ao início das infor- mações do SIH-SUS em CD-ROM e ao descredenciamento da Clínica Santa Genoveva (MS, 1998b).

A seleção dos hospitais de referência foi feita a partir das internações, remuneradas pelo SUS, que preenchiam os critérios de comparabilidade adotados neste trabalho. Estes critérios foram: 1) diagnóstico da internação no mesmo grupo de patologias, correspondentes a 90\% das internações realizadas na Clínica Santa Genoveva, ou seja, efeitos tardios de doença cerebrovascular (CID-9: 438), arteriosclerose (CID-9: 440), outros tipos de desnutrição proteico-calórica não especificada (CID-9: 263), oclusão das artérias cerebrais (CID-9: 434), diabetes mellitus (CID-9: 250), efeitos tardios de lesões e traumatismos osteomusculares e/ou tecido conjuntivo (CID-9: 905) e insuficiência cardíaca (CID-9: 428) (Tabela 1); 2) internações cujo procedimento principal fosse atendimento a paciente fora de possibilidade terapêutica (FPT), código 85100030 da tabela de procedimentos do SIH-SUS, as quais correspondiam ao total das internações realizadas na Clínica Santa Genoveva. Este último critério é justificável porque, no âmbito do SUS, o procedimento principal define os procedimentos cobertos pelo sistema durante a internação, bem como a remuneração paga ao hospital. Para a obtenção de indicadores estáveis foram incluídos no estudo apenas os hospitais com $500 \mathrm{ou}$ mais internações/ano que preenchessem os critérios acima mencionados.

O número da AIH é único para cada paciente durante a mesma internação, não sendo reutilizado após a saída do hospital. Em casos de internações prolongadas (AIH de identificação 5), a mesma AIH pode ser computada várias vezes para fins de faturamento. Após 180 dias de internação, havendo necessidade de o paciente continuar internado, o hospital solicita nova AIH (MS, 1998b). No presente trabalho, para evitar que a mesma AIH fosse computada mais de uma vez em mesmo mês e ano, foi feita a verificação de todo o banco de dados, de forma a eliminar duplicações. Assim, somente ocorreria a dupla contagem do paciente no período (mês e ano) naqueles casos em que o paciente ficasse internado por mais de 180 dias e nova AIH fosse emitida no mesmo mês. Desta forma tornou-se possível estimar o número de pacientes/mês durante o período estudado.

As seguintes informações relativas às internações foram consideradas neste trabalho: idade (<60anos; 60-69anos; 70-79anos; $\geq 80$ anos), sexo (masculino ou feminino), tipo de $\mathrm{AIH}$ (identificação 1 e identificação 5), motivo da saída (óbito ou não), data do óbito (mês e ano 
Diagnóstico principal que justificou a internação dos pacientes da Clínica Santa Genoveva, Rio de Janeiro, segundo a condição de saída (óbito ou não). Janeiro de 1993 a maio de 1996.

\begin{tabular}{|c|c|c|c|c|}
\hline CID 9 & Descrição & $\begin{array}{l}\text { Óbito } \\
n \%\end{array}$ & $\begin{array}{l}\text { Não óbito } \\
n \text { \% }\end{array}$ & $\begin{array}{l}\text { Total } \\
\mathrm{n} \%\end{array}$ \\
\hline 438 & $\begin{array}{l}\text { Efeitos tardios } \\
\text { doença cerebrovascular }\end{array}$ & $458(54,9)$ & $2.025(51,6)$ & $2.483(52,1)$ \\
\hline 440 & Arteriosclerose & $131(15,7)$ & $625(15,9)$ & $756(15,9)$ \\
\hline 263 & $\begin{array}{l}\text { Outros tipos desnutrição } \\
\text { proteico-calórica não especificada }\end{array}$ & $53(6,4)$ & $171(4,4)$ & $224(4,7)$ \\
\hline 434 & Oclusão das artérias cerebrais & $42(5,0)$ & $179(4,6)$ & $221(4,6)$ \\
\hline 250 & Diabetes Mellitus & $34(4,1)$ & $106(2,7)$ & $140(2,9)$ \\
\hline 905 & $\begin{array}{l}\text { Efeitos tardios de lesões e } \\
\text { traumatismos osteomusculares } \\
\text { e/ou tecido conjuntivo }\end{array}$ & $23(2,8)$ & $367(9,3)$ & $390(8,2)$ \\
\hline \multirow[t]{3}{*}{428} & Insuficiência cardíaca & $18(2,2)$ & $57(1,5)$ & $75(1,6)$ \\
\hline & Outras causas & $75(9,0)$ & $398(10,1)$ & $473(9,9)$ \\
\hline & Total & $834(100,0)$ & $3.928(100,0)$ & $4.762(100,0)$ \\
\hline
\end{tabular}

Qui-quadrado $_{(d f=7)}=51,67, p<0,001$.

de ocorrência), diagnóstico principal da internação (CID 9; 3 dígitos) e procedimento principal (tabela de procedimentos do SIH-SUS). As AIH de identificação 5 muitas vezes não tinham sido preenchidas nos campos referentes à idade e ao sexo. Nestes casos, as informações foram obtidas na AIH de identificação 1, que corresponde ao início da internação do paciente.

Neste estudo, as taxas mensais de mortalidade hospitalar foram avaliadas tomando-se, como numerador, os óbitos ocorridos entre os pacientes internados pelo SUS e, como denominador, o total de pacientes internados pelo SUS naquele mês, incluindo, portanto, as internações que se iniciaram no mês e aquelas começadas em meses anteriores.

\section{Análise dos dados}

Para comparação entre proporções foi utilizado o teste do Qui-quadrado (Fleiss, 1981). As razões das taxas mensais de mortalidade brutas ou ajustadas por idade e sexo (riscos relativos e intervalos de confiança ao nível de 95\%; RR e IC 95\%, respectivamente) foram calculadas através da regressão de Poisson (Breslow \& Day, 1987).

Quando a taxa de mortalidade mensal na Clínica Santa Genoveva foi comparada à dos hospitais de referência, os riscos relativos ajustados por idade e sexo foram estimados para todos os meses estudados, exceto para janeiro, fevereiro, março e abril de 1993. Riscos relativos brutos foram utilizados para esses quatro meses, porque a perda diferencial de informação sobre idade e sexo neste período foi grande e isso poderia introduzir viéses na interpretação dos resultados.

A análise dos dados foi feita mediante a utilização dos pacotes Epi-Info (Dean et al., 1994) e Stata (Stata Corporation, 1997).

\section{Resultados}

Foram identificadas 4.762 Autorizações de Internações Hospitalares (AIH) de pacientes internados pelo SUS na Clínica Santa Genoveva durante o período investigado. Isto corresponde a um total de 13.240 pacientes/mês internados, com média mensal de 323 pacientes. Trinta e quatro por cento dos pacientes/mês observados (4.530) correspondiam a AIH de identificação 1 (inicial) e 66\% (8.710) a AIH de identificação 5 (longa permanência).

As informações referentes a idade e sexo foram obtidas para $94 \%$ dos pacientes. As perdas 
de informações relativas a idade e sexo concentraram-se nos quatro primeiros meses de 1993 (40\% das internações no período). Nos anos seguintes, as perdas de informações destas variáveis estiveram abaixo de $5 \%$. Para os 4.484 pacientes com informação disponível para sexo e idade, $72 \%$ eram do sexo masculino e $28 \%$ do sexo feminino. As mulheres eram mais velhas que os homens (mediana da idade $=72$ e 65 anos; percentil $25=61$ e 55 , percentil $75=$ 80 e 75 , respectivamente).

Durante os 41 meses estudados ocorreram 834 óbitos na Clínica Santa Genoveva, correspondendo à taxa bruta de mortalidade hospitalar igual a 63 por 1.000 pacientes/mês. A taxa de mortalidade aumentou progressivamente com a idade: $44 / 1.000$ pacientes/mês nos menores de 60 anos (classe de referência), 46/1.000 naqueles com 60-69 anos (RR = 1,05; IC-95\% = 0,84-1,30); 73/1.000 naqueles com 70-79 anos $(\mathrm{RR}=1,65 ; \mathrm{IC}-95 \%=1,35-2,01)$ e $106 / 1.000$ nos pacientes com mais de 80 anos de idade $(\mathrm{RR}=2,42$; IC-95\% = 1,99, 2,94). A taxa bruta de mortalidade hospitalar no período foi maior no sexo feminino em comparação ao masculino (87/1.000 e 53/1.000 pacientes mês, respectivamente); a taxa de mortalidade entre os pacientes sem informação quanto ao sexo (65/1.000 pacientes mês) foi semelhante à taxa bruta global do período. A diferença entre sexos persistiu após o ajustamento pela idade $(\mathrm{RR}=1,5$; $\mathrm{IC}$ $95 \%=1,3-1,7)$.

Na Tabela 1 são mostrados os diagnósticos principais que justificaram a internação dos

Tabela 2

Número de óbitos entre pacientes internados na Clínica Santa Genoveva, Rio de Janeiro, segundo o mês e o ano de ocorrência. Janeiro de 1993 a maio de 1996.

\begin{tabular}{lrrrr}
\hline Meses & \multicolumn{5}{c}{ Número de óbitos } \\
& 1993 & 1994 & 1995 & 1996 \\
\hline Janeiro & 47 & 16 & 14 & 32 \\
Fevereiro & 20 & 10 & 10 & 18 \\
Março & 20 & 23 & 22 & 28 \\
Abril & 14 & 18 & 7 & 28 \\
Maio & 26 & 17 & 5 & 50 \\
Junho & 30 & 27 & 9 & - \\
Julho & 21 & 21 & 5 & - \\
Agosto & 32 & 31 & 16 & - \\
Setembro & 19 & 27 & 10 & - \\
Outubro & 19 & 15 & 12 & - \\
Novembro & 15 & 17 & 23 & - \\
Dezembro & 14 & 10 & 36 & - \\
Total & 277 & 232 & 169 & 156 \\
\hline
\end{tabular}

pacientes na Clínica Santa Genoveva segundo a condição de saída (óbito ou não). A causa mais freqüente de internação nos dois grupos foi efeito tardio de doença cerebrovascular (CID-9 438) (54,9\% e $52,1 \%$, respectivamente). Houve diferença significante $(\mathrm{p}<0,001)$ na distribuição de óbitos e não óbitos quanto ao diagnóstico. A diferença se concentrou nos casos com diagnóstico de efeitos tardios de lesões e traumatismos osteomusculares elou tecido conjuntivo (CID-9 905) (2,8\% entre os que faleceram e 9,3\% entre os que não faleceram).

O número de óbitos mensais entre pacientes internados na Clínica Santa Genoveva variou entre 5 e 50 durante o período estudado. Os picos na mortalidade foram observados nos meses de janeiro de 1993 (47 óbitos) e maio de 1996 (50 óbitos) (Tabela 2).

Na Figura 1 estão apresentadas as taxas brutas mensais de mortalidade hospitalar na Clínica Santa Genoveva e para o conjunto dos hospitais de referência. No mês imediatamente anterior ao descredenciamento da clínica (maio de 1996), verificou-se taxa de mortalidade igual a 142,9 por 1.000 pacientes. Taxa razoavelmente semelhante foi observada na mesma clínica três anos antes (131,7 por 1.000 pacientes em janeiro de 1993). As taxas de mortalidade na Clínica Santa Genoveva e nos hospitais de referência apresentaram grande variação no período considerado. Cabe ressaltar, entretanto, que as taxas brutas de mortalidade observadas na Clínica Santa Genoveva foram superiores às observadas nos hospitais de referência na maioria dos meses estudados (Figura 1).

A Figura 2 apresenta os riscos relativos para óbito na Clínica Santa Genoveva em comparação aos hospitais de referência. Os maiores riscos relativos, quando o risco de morrer mostrou-se duas vezes maior entre os pacientes da clínica, foram notados em janeiro de 1993 $\left(\mathrm{RR}_{\text {bruto }}=2,23\right.$; IC-95\% = 1,56-3,14), agosto de $1993\left(\mathrm{RR}_{\text {ajustado }}=1,99 ; \mathrm{IC}-95 \%=1,34-2,94\right)$, dezembro de $1995\left(\mathrm{RR}_{\text {ajustado }}=2,20\right.$; IC-95\% = $1,45-3,34)$ e maio de $1996\left(\mathrm{RR}_{\text {ajustado }}=2,73\right.$; $\mathrm{IC}$ $95 \%=1,88-3,95)$. O risco de morrer na clínica foi superior ao dos hospitais de referência em 10 dos 41 meses investigados (valores de RR e limites inferiores do IC-95\% > 1). Somente em um dos meses estudados (julho de 1995), o risco de morrer entre os pacientes da Clínica Santa Genoveva foi menor do que aquele observado nos hospitais de referência $\left(R_{\text {ajustado }}=0,29\right.$; IC-95\% $=0,12-0,73$ ) 


\section{Figura 1}

Taxa de mortalidade (por 1000 pacientes internados) entre pacientes da Clínica Santa Genoveva, Rio de Janeiro e entre os pacientes dos hospitais de referência (janeiro, 1993 - maio, 1996).

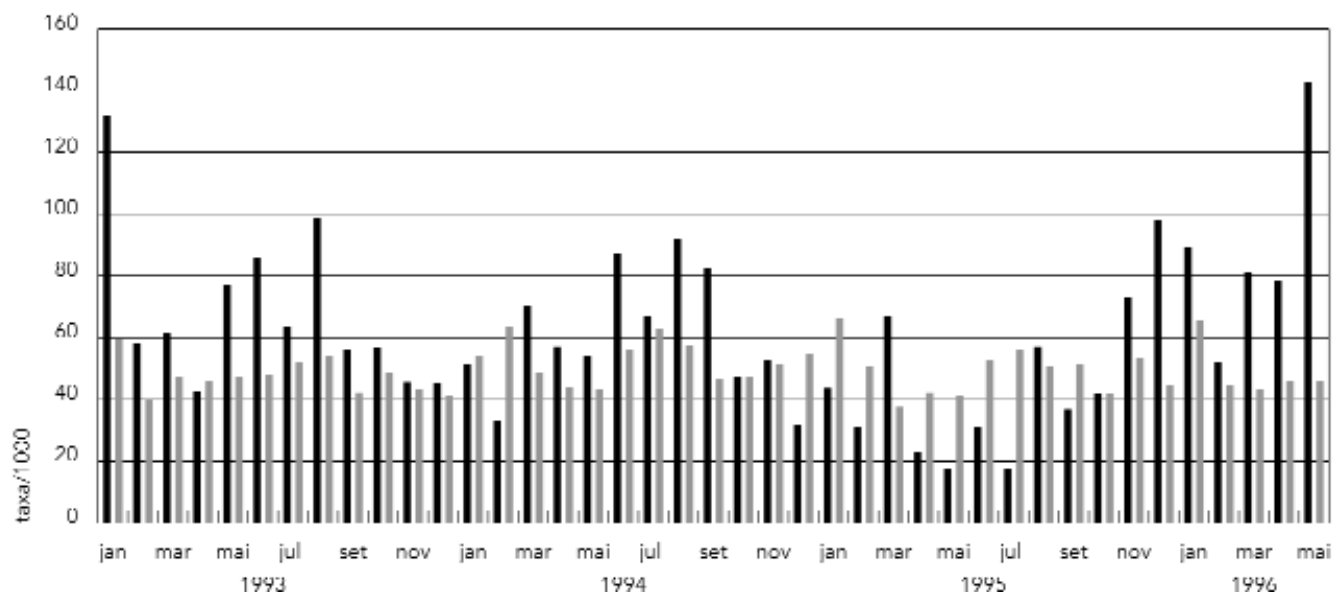

Sianla Genouveria

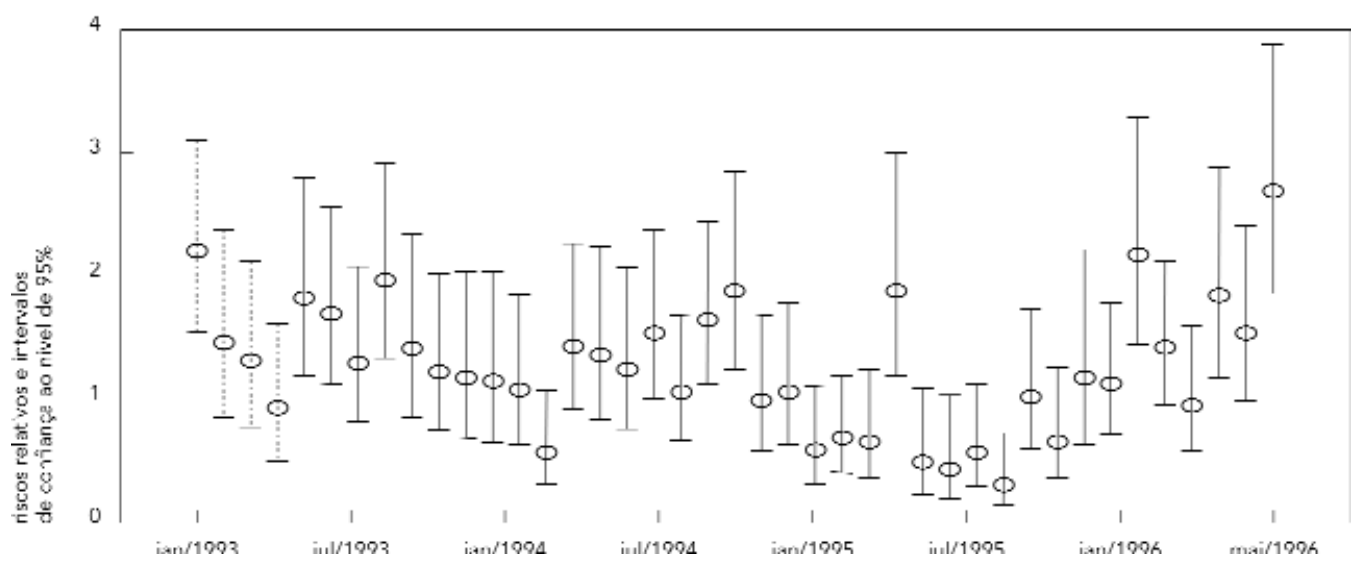




\section{Discussão}

Os resultados deste trabalho evidenciam que os óbitos ocorridos na Clínica Santa Genoveva em maio de 1996 não constituíram fato isolado, mas sim tendência da clínica durante o período investigado. Esta tendência foi consistentemente observada na (1) verificação da freqüência mensal de óbitos, (2) na verificação das taxas brutas mensais de mortalidade e (3) nas comparações entre as taxas mensais de mortalidade observadas na Clínica Santa Genoveva e aquelas dos hospitais de referência.

A taxa de mortalidade ajustada por características dos pacientes e dos hospitais tem sido usada, há mais de 20 anos, para monitorar a qualidade dos hospitais nos Estados Unidos (Roemer et al., 1968; DesHarnais et al., 1988), não obstante algumas críticas apresentadas quanto a este indicador (Hofer \& Hayward, 1996). A utilização, no presente trabalho, das taxas brutas de mortalidade foi suficiente para confirmar os resultados da prospecção do número mensal de óbitos, mas a introdução de um parâmetro externo de comparação reforçou e deu maior consistência aos achados descritivos.

Quanto à adequação da referência utilizada para comparação externa, embora ainda não aproveitada no meio da saúde, teve, como referência teórica, a construção dos Diagnosis Related Groups (DRGs) (Noronha et al., 1991). Os critérios aqui adotados foram baseados no caráter público da internação, semelhança de diagnósticos, procedimento principal e volume de atendimentos realizados pelos hospitais, respeitando as principais premissas de comparabilidade propostas para este tipo de estudo (Roemer et al., 1968; DesHarnais et al., 1988; Flood, 1990).

Uma questão relevante a ser discutida em investigação que se funda em dados secundários é a qualidade da informação empregada. Veras \& Martins (1994) estudaram amostra das internações hospitalares públicas de pacientes agudos no Rio de Janeiro em 1982. Os autores encontraram elevada concordância entre as informações obtidas a partir dos prontuários médicos e aquelas presentes no formulário da $\mathrm{AIH}$ para os dados de faixa etária, sexo, óbito hospitalar e diagnóstico de internação ao nível de três dígitos. Apesar dessas variáveis serem as mesmas utilizadas no presente trabalho, as AIH aqui estudadas referem-se a pacientes de longa permanência e idosos, e é possível que a confiabilidade neste tipo de serviço difira daquela observada por Veras \& Martins (1994).

O presente trabalho é exemplo de como o uso de dados secundários da atenção médica pode ser útil para investigações epidemiológicas e avaliação de serviços de saúde no Brasil. Flood (1990) defende os estudos baseados em dados secundários, em comparação aos ensaios clínicos, para investigações de serviços de saúde. Tais estudos são eticamente aceitáveis e metodologicamente adequados para a avaliação da efetividade da assistência hospitalar, uma vez que refletem o processo real e cotidiano de atenção aos pacientes.

Em resumo, os resultados mostram que, no episódio da Clínica Santa Genoveva, a utilização dos dados do SIH-SUS poderia ter antecipado as investigações dos órgãos competentes, de modo a evitar o excesso de mortalidade em idosos, identificado apenas em meados de 1996. No momento em que o Brasil vivencia crescimento progressivo da população idosa, o caso da Clínica Santa Genoveva chama a atenção para a vulnerabilidade dessa população. O SIHSUS oferece dados importantes para o monitoramento e avaliação da assistência hospitalar prestada pela rede conveniada. Como não há motivos para acreditar que o episódio da mencionada clínica seja fato isolado, considera-se que este tipo de investigação é fundamental para estabelecer métodos de monitoramento e contribuir para a construção de indicadores de avaliação da qualidade da atenção à saúde. 


\section{Referências}

BRESLOW, N. E. \& DAY, N. E., 1987. Statistical Methods in Cancer Research: The Analysis of Cohort Studies. IARC Scientific Publications n. 82. Lyon: International Agency for Research on Cancer.

DEAN, A. G.; DEAN, J. A.; COLOUMBIER, D.; BRENDEL, K. A.; SMITH, D. C.; BURTON, A. H.; DICKER, R. C.; SULLIVEN, K.; TAGAN, R. F. \& ARNET, T. G., 1994. Epi Info version 6.0: A Word Processing Database and Statistics Program for Epidemiology on Microcomputers. Atlanta: Centers for Disease Control and Prevention.

DESHARNAIS, S. I.; CHESNEY, J. D.; WROSBLEWSKI, R. T.; FLEMING, S. T. \& McMAHON Jr., L. F., 1988. The risk-adjust mortality index. A new measure of hospital performance. Medical Care, 26:1129-1148.

FLEISS, J. L., 1981. Statistical Methods for Rates and Proportions. 2nd Ed. New York: John Wiley.

FLOOD, A. B., 1990. Peaks and pits of using large data bases to measure quality of care. International Journal of Technology Assessment in Health Care, 6: 253-262.

FOLHA DE SÃO PAULO, 1996. O caos na saúde pública. Folha de São Paulo, São Paulo, 22 nov., Caderno 3, p. 1.

HOFER, T. P. \& HAYWARD, R. A., 1996. Identifying poorquality hospital. Can hospital mortality rates detect quality problems for medical diagnoses? Medical Care, 34:737-753.
MS (Ministério da Saúde), 1998a. Manual da AIH. In: Movimento de Autorização de Internações Hospitalares, 1993-1998. CD-ROM. Brasília: Departamento de Informática do SUS, Ministério da Saúde.

MS (Ministério da Saúde), 1998b. Movimento de Autorização de Internações Hospitalares, 1993-1998. CDROM. Brasília: Departamento de Informática do SUS, Ministério da Saúde.

NORONHA, M. F.; VERAS, C. T.; LEITE, I. C.; MARTINS, M. S.; BRAGA NETO, F. \& SILVER, L., 1991. O desenvolvimento dos "Diagnosis Related Groups" DRGs. Metodologia de classificação de pacientes hospitalares. Revista de Saúde Pública, 25:198-208.

ROEMER, M. I.; MOUSTAFA, A. T. \& HOPKINS, C. E., 1968. A proposed hospital quality index: Hospital death rates adjusted for case severity. Health Services Research, 3:96-118.

STATA CORPORATION, 1997. Stata Statistical Software: Release 5.0. College Station: Stata Corporation.

VERAS, C. M. T. \& MARTINS, M. S., 1994. A confiabilidade dos dados nos formulários de autorização de internação hospitalar (AIH), Rio de Janeiro, Brasil. Cadernos de Saúde Pública, 10:339-355. 